This material is published in the open archive of Mid Sweden University DIVA http://miun.diva-portal.org to ensure timely dissemination of scholarly and technical work. Copyright and all rights therein are retained by authors or by other copyright holders. All persons copying this information are expected to adhere to the terms and constraints invoked by each author's copyright. In most cases, these works may not be reposted without the explicit permission of the copyright holder.

Yu, K.; Gidlund, M.; Åkerberg, J., and Björkman, M. "Performance Evaluations and Measurements of the REALFLOW Routing Protocol in Wireless Industrial Networks," in IEEE Transactions on Industrial Informatics, vol. 13, no. 3, pp. 1410-1420, June 2017.

https://doi.org/10.1109/TII.2016.2587842

(C)2016

IEEE. Personal use of this material is permitted. However, permission to reprint/republish this material for advertising or promotional purposes or for creating new collective works for resale or redistribution to servers or lists, or to reuse any copyrighted component of this work in other works must be obtained from the IEEE. 


\title{
Performance Evaluations and Measurements of the REALFLOW Routing Protocol in Wireless Industrial Networks
}

\author{
Kan Yu, Mikael Gidlund, Johan Åkerberg, Senior Member, IEEE, and Mats Björkman
}

\begin{abstract}
Industrial wireless sensor and actuator networks (IWSANs) offer significant advantages to industrial automation. However, high-reliability demands and hard communication deadlines pose challenges to its practical applications. To achieve this goal, flooding is considered as a promising approach due to multipath diversity and simplicity. In this paper, an enhanced version of REALFLOW, a flooding-based routing protocol for IWSANs is presented. Compared to the original REALFLOW, network management and network stability are improved. REALFLOW is compared with four other flooding protocols via simulations. The simulation results show that REALFLOW has better performance in terms of reliability and consecutive transmission errors when considering deadlines. Compared with normal flooding, REALFLOW achieves comparable reliability performance with decreased redundancy. Measurements from a prototype implementation conducted in an industrial manufacturing workshop reveal that high-reliability and low-application failure rates can be achieved, giving more confidence in providing reliable wireless sensing and actuating for industrial automation.
\end{abstract}

Index Terms_-Industrial wireless sensor network (WSN), measurement, multipath, reliability, routing.

\section{INTRODUCTION}

I NDUSTRIAL wireless sensor and actuator networks (IWSANs) have been increasingly applied in industrial automation and are able to serve a number of purposes, such as supervision and control. Requirements from industrial automation at the machine level are usually more stringent than those from traditional applications. First, high reliability should be guaranteed [1]; otherwise, the quality of control may be degraded. Moreover, industrial control systems are usually designed to be tolerant of single communication errors. However, if a pro-

Manuscript received July 7, 2015; revised December 17, 2015 and March 29, 2016; accepted May 17, 2016. Date of publication July 7, 2016; date of current version June 1, 2017. Paper no. TII-15-1128.R2. (Corresponding author: K. Yu.)

K. Yu and M. Björkman are with the School of Innovation, Design and Engineering, Mälardalen University, Västerås 721 23, Sweden (e-mail: kan.yu@mdh.se; mats.bjorkman@mdh.se).

M. Gidlund is with the Mid Sweden University, Sundsvall 85170 , Sweden (e-mail: mikael.gidlund@miun.se).

J. Åkerberg is with the ABB Corporate Research, Västerås 721 78, Sweden (e-mail: johan.akerberg@se.abb.com).

Color versions of one or more of the figures in this paper are available online at http://ieeexplore.ieee.org.

Digital Object Identifier 10.1109/TII.2016.2587842 cess experiences consecutive communication errors, it may be terminated for safety reasons and then the system availability suffers. As a result, it may take up to several hours to achieve full production rate again, which may lead to significant production losses [1].

Currently, monitoring via IWSANs for certain industrial applications has been demonstrated [2]. However, their applicability in reality is still in the development stage. A number of research efforts have been taken in this field, but to provide reliable and timely communication via IWSANs for mission critical industrial applications remain challenges. Thus, this paper aims for narrowing the gap between existing research and industrial applications. One approach is to apply an appropriate routing protocol. For instance, flooding is more reliable than unicasting due to the one-to-many transmission property. Compared with multicasting, flooding behaves simply and takes inherent advantage of wireless communication. From the bandwidth usage perspective, there is no difference whether a packet is transmitted to one or several neighbors wirelessly. It has been shown that flooding might be one feasible alternative in mission-critical wireless sensor networks (WSNs) [3].

Admittedly, low-energy consumption should be and has already been concerned [4]-[6]. However, there is a tradeoff between reliability and energy consumption [2]. Approaches to reduce transmission failure, such as coding and retransmission, may require additional energy. In many industrial applications, reducing energy consumption should not be prioritized as high as reliability, since if IWSANs are unreliable, minimizing energy consumption will be in vain. For instance, in [6], a routing and topology control algorithm is proposed to reduce energy consumption, but reliability and real-time performance is not fully considered in this study.

To provide reliable and deadline-constrained communication, a flooding-based mechanism is applied for routing. Different from traditional routing protocols, a flooding-based routing has several advantages. First, multipath diversity can be achieved. The second advantage is called wireless broadcast advantage (WBA) [7]. Specifically, due to the broadcast nature of wireless medium, all adjacent nodes of a transmitting node may overhear the packets, so packet forwarding can be more flexible. Finally, because of broadcasting behavior, link level acknowledgment (ACK) can be avoided to shorten the transmission latency. One may argue that without ACK and retransmissions reliable 
communication cannot be guaranteed. However, compared with the automatic repeat request (ARQ) method, the flooding-based mechanism allows packets to be sent instantaneously via multiple paths rather than one path at different time. For ARQ, the number of retries is usually defined to prevent excessive retransmissions. Therefore, if the number of multiple paths equals to the number of retries, the flooding-based mechanism achieves similar level of reliability to ARQ, but with shorter delay.

In this paper, an enhanced version of the flooding-based routing protocol (REALFLOW) based on previous research [8] is presented. To fulfill the strict requirements of mission-critical industrial applications, we applied an improved network discovering method and data forwarding criteria. Thus, reliable and timely transmissions can still be achieved. Compared with the previous solution, several improvements are made to adapt our protocol to real implementation and real industrial environments. For instance, the command distribution method is proposed and the routing maintenance approach is modified to achieve efficient network management and network stability in reality. To evaluate the performance, the enhanced REALFLOW is compared with four other flooding protocols via simulation in terms of reliability, availability, and efficiency. The results show that our proposed protocol performs better than other advanced flooding-based protocols with higher packet delivery ratio and shorter length of consecutive failures given packet deadlines. Even compared with normal flooding, our proposed protocol still exhibits comparable performance with decreased redundancy. To verify the correctness in real environments, an IWSAN prototype is built and the protocol stack is implemented. Measurements conducted in a manufacturing workshop indicate that REALFLOW achieves reliable communication with short lengths of consecutive errors, which gives more confidence in fulfilling the requirements of applying wireless sensing and control for industrial automation. The contributions of this paper are summarized as follows.

1) Present the enhanced REALFLOW protocol for reliable and deadline constrained communication.

2) Conduct simulations of REALFLOW and other floodingbased routing protocols for comparison.

3) Conduct measurements in a real industrial environment based on our implementation and analyze the measurement results according to the industrial requirements.

The rest of this paper is organized as follows: Section II presents previous work on routing protocols in wireless networks. In Section III, the protocol REALFLOW is outlined. The simulation settings and result analysis are described in Section IV, followed by the implementation and measurement in Section V. Finally, this paper is concluded in Section VI.

\section{RELATED WORK}

Multipath diversity has been recognized as an efficient property to improve reliability in WSNs. A number of multipath routing protocols for WSNs have been extensively introduced in the survey paper [9]. Over the last few years, more multipath routing protocols for WSNs were published. Although the reliability performance can be greatly improved by this set of protocols, how to meet the packet deadline is not fully consid- ered. Therefore, even if packets arrive at the destination reliably, if the deadline is missed, application failures still occur. Moreover, multipath routing protocols usually require more overhead to maintain all links information, such as searching the shortest paths.

A number of recent publications on routing protocols for deterministic delay in IWSNs can be found. Quang and Kim [10] proposed a two-hop neighbor information-based gradient routing. According to their simulation results, high deadline delivery success ratio can be achieved. A similar two-hop neighborhood information-based geographic routing protocol is proposed in [11] by mapping packet deadline to the velocity. Quang and Kim [12] presented a cluster-based throughput-aware routing protocol for IWSANs to decrease latency. One major advantage of this protocol is that synchronization of the whole network is not required, which is suitable for a large-scale network, but extra equipped cluster heads are needed, which increases the cost of deployment. All these protocols aim for real-time performance in IWSNs, but high reliability is not their main goal. Another limitation of these protocols is that only uplink from field devices to the sink is supported.

Several new protocols were proposed for both high-reliability and real-time performance. Niu et al. [13] presented a reliable reactive routing enhancement (R3E) to enhance existing reactive routing protocols by utilizing the local path diversity. The main advantage is that data packets can be greedily forwarded toward destinations through nodes' cooperation without utilizing location information, but R3E as a reactive protocol has higher latency than proactive protocols. Heo et al. [14] proposed EARQ, where next hop selections are based on the estimations of energy consumption, reliability, and deadlines. Han et al. [15] also proposed a reliable graph routing scheme. In [16], Zou et al. pointed out that an increasing number of paths achieve high reliability when links become burstier. Oh et al. [17] also proposed a reliable and real-time protocol for WSNs by forwarding packets via disjoint multipath with geographical parallel track concept, so additional geographical is required. EchoRing is proposed in [18] as wireless token-passing protocol for high reliability and low latency, but it is designed for decentralized architecture.

Different from the protocols above, flooding is also a classical communication approach. Previously flooding is more often used for network management, such as route discovery. However, flooding can also be applied as a data dissemination method. Although a flooding-based protocol has several advantages mentioned previously, the drawbacks cannot be neglected. The biggest concern is broadcast storm. Specifically, normal flooding may produce excessive amounts of redundant traffic. The second drawback is nondirectional transmission. Since packets are forwarded to all neighbors, unnecessary packet forwarding may occur. Therefore, a number of researches on flooding-based routing have been done. Gossiping [19] avoids the inherent drawbacks of normal flooding, but long latency limits their usages. Directional flooding proposed in [20] solves the previous problem, but to control the flooding area is a challenge. Yen et al. [21] proposed a new flooding approach by combining gossiping with directional flooding, aiming for balanced performance. Location-based flooding is proposed in [22] 
to achieve a lightweight flooding method, but obtaining location information is not always possible in reality. To the best of our knowledge, most flooding-based routing protocols can only support uplink transmission from a node to a sink. A little research effort on downlink transmission can be found. Ferrari et al. in [23] proposed Glossy, a novel flooding architecture for WSNs, which can achieve low latency packet transmissions and fast time synchronization. Different from previous flooding protocols, Glossy is a one-to-many transmission pattern, which is not suitable for one-to-one transmission scenarios. Another advanced routing protocol, named cooperative routing protocol [7], also takes advantage of this merit, but floodingbased routing protocols are still considered to be simpler and more suitable for centralized network architectures, compared with cooperative routing protocols, as well as those multipath routing protocols.

Compared with above nonflooding-based routing protocol, our proposed enhanced REALFLOW has all the advantages of the flooding mechanism, such as multipath diversity, WBA and no need for ACK, so both high reliability and short latency can be achieved. Moreover, the overhead of the network management of our protocol is also lower. The reason is that packet transmissions by broadcasting do not need to follow specific paths, so it is unnecessary to frequently update specific information of all links. With the enhancement of REALFLOW, the cost of network management will be even lower. Compared with those flooding-based routing protocols, the major advantage of our protocol is to support both reliable and deadline constrained uplink and downlink transmissions, whereas most flooding-based routing protocol can only support one direction. Another advantage of our proposed protocol is that location or geographical information is not required, which decease the complexity and make our protocol suitable for more application scenarios. Finally, our proposed protocol can be easily implemented in real devices. Both simulation results and measurement results in real industrial environments showed that our protocol can fulfill those strict requirements from mission-critical industrial applications, which will be introduced later in this paper.

\section{REALFLOW IN IWSANS}

This section presents the details of the enhanced REALFLOW. The proposed protocol is adapted with several improvements, mainly from three aspects: 1) the parent selection process; 2) routing for unicasting command packets; 3) the scheduling distribution approach. First, the system model is described. Then, REALFLOW is outlined from two perspectives: the route establishment/maintenance method and packet forwarding criteria, in Sections III-B and III-C, respectively. Finally, an example of enhanced REALFLOW is demonstrated.

\section{A. System Model}

A centralized architecture is usually applied in an IWSAN. For simplicity, two basic types of devices are used in this study: 1) gateway: it is responsible for managing the network activities, including scheduling, topology maintenance, time synchronization management, etc. It also bridges the wireless network to the

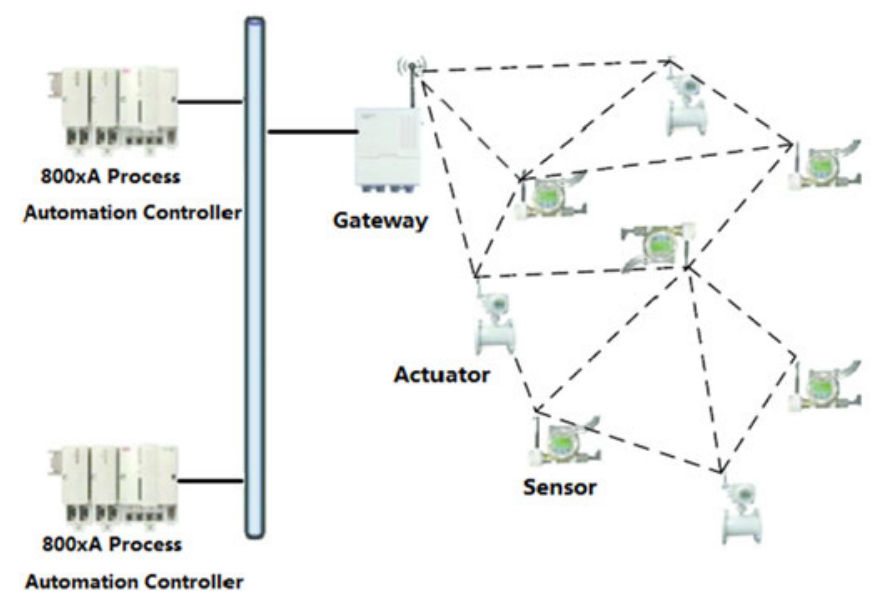

Fig. 1. Typical IWSAN topology.

TABLE I

INFORMATION IN AN LIST-UPDATE MESSAGE

\begin{tabular}{ll}
\hline \hline Notation & \multicolumn{1}{c}{ Description } \\
\hline$r_{\mathrm{pkt}}$ & the accumulated RSSI value \\
$h_{\mathrm{pkt}}$ & the hop number from the gateway \\
$A_{\mathrm{prev}}$ & the previous node address \\
$A_{\mathrm{src}}$ & the source node address \\
\hline \hline
\end{tabular}

TABLE II

REALFLOW INFORMATION IN AN NODE

\begin{tabular}{ll}
\hline \hline Notation & \multicolumn{1}{c}{ Description } \\
\hline $\mathbb{V}$ & the history of the received list-update messages \\
$K_{\max }$ & the maximum allowed parent nodes \\
$\mathbb{L}$ & the related node list \\
\hline \hline
\end{tabular}

control system; 2) field devices: these devices usually consist of sensor nodes and actuator nodes.

A simplified IWSAN is shown in Fig. 1. From the figure, programmable logic controllers are connected to the gateway. All sensor nodes in the network periodically send the latest sensing data to the control system via the gateway. After data have been processed, the output values are obtained by the gateway and distributed to corresponding actuator nodes.

\section{B. Route Establishment and Maintenance}

To discover and maintain routes, the gateway periodically broadcasts network discovery messages named list-update message. The purpose is to assist nodes in finding and identifying their parent nodes. Each message contains important information summarized in Table I. Each node also maintain several important values, which is summarized in Table II.

When a node receives a list-update message, if this message is not seen before, the received signal strength indicator (RSSI) is obtained from the radio chip and added to $r_{\mathrm{pkt}}$ of this message. $h_{\mathrm{pkt}}$ is also increased by one. Then, $A_{\mathrm{prev}}, r_{\mathrm{pkt}}$, and $h_{\mathrm{pkt}}$ are stored in a set $\mathbb{V}$. After $A_{\text {prev }}$ is replaced with the current node address, the list-update message is rebroadcasted.

Afterward, each node broadcasts a response named listresponse message. $K_{\max }$, as a predefined value, specifies the 
maximum number of parents that a node can choose from $\mathbb{V}$. A larger $K_{\max }$ indicates more transmission paths, but at the cost of network resources. Thus, the selection of $K_{\max }$ should be based on specific scenarios. The parent nodes are selected from $\mathbb{V}$ according to the channel quality, which is indicated by $r_{\mathrm{pkt}}$ and $h_{\mathrm{pkt}}$ and reported to the gateway in a list-response message. A related node table $\mathbb{L}$ should be generated during forwarding of list-response messages. As a key element of REALFLOW, $\mathbb{L}$ is used for constraining the flooding area and providing directional flooding. To support both data and command packet routing, a list-response message contains both the previous forwarding node address and its selected parents. When a relay node receives a list-response message, if the current node is the parent of the previous forwarding node, the source node of this message is defined as a related node of the current node. Both $A_{\text {src }}$ and $A_{\text {prev }}$ are inserted into $\mathbb{L}$. After receiving responses from all nodes, the gateway obtains the latest network topology.

As industrial environments are harsh and dynamic, parent nodes for each node may change frequently. Due to the centralized management and TDMA scheme, once parent nodes change, the gateway has to send new scheduling commands to all relevant nodes. Frequent topology changes will cause excessive network management overhead, which might result in network instability. More specifically, if one node in the network needs to change its parents, the network topology will be changed. Then, other nodes, even the whole network, need to be rescheduled. Although by using certain optimized scheduling algorithms, unrelated nodes may not have to change their schedule. However, this cannot be guaranteed. An effective solution is to avoid unnecessary parent changes. Thus, the parent node updating approach is improved in this study. When a new round of maintenance begins, each node will preserve its previous parent nodes. During the selection of parent nodes, each node will first check if the previous parents exist in the latest $\mathbb{V}$. If the previous parents are found and their corresponding $h_{\mathrm{pkt}}$ is the same as the current one, the parent nodes will be reused; otherwise, new parents will be chosen. This enhancement effectively prevents frequent topology variations and improves overall performance.

Due to TDMA applied on the MAC layer for deterministic communication, each node should be assigned with dedicated timeslots. To make all nodes agree on the latest scheduling, a two-phase commit protocol is used. First, the gateway sends scheduling requests to all nodes, containing the latest decision. When the decisions are received, all nodes should reply scheduling responses to the gateway. Once the gateway receives all responses, scheduling confirmation messages are sent to all nodes, containing the activation time of the new schedule. After receiving confirmation messages, all nodes will activate the new schedule at the specified time. If any failures occur during the schedule distribution, the whole process will restart again. Routing for scheduling messages can be the same as routing for date messages, which will be described in the next section.

\section{Packet Forwarding Criteria}

Now the packet forwarding criteria of REALFLOW can be formulated. The first forwarding criterion is that if a node re- ceives an uplink packet with the source address $A_{u}$ or a downlink packet with destination address $A_{d}$, and $A_{u} / A_{d}$ can be found in the local $\mathbb{L}$, this packet is allowed to be forwarded. This criterion constrains the flooding area to relevant nodes.

To avoid duplicated packet forwarding, each packet contains a sequence number $s_{\mathrm{pkt}}$. Along with the source address $A_{\mathrm{src}}$, a unique pair $P$ can be constructed, where $P=\left(s_{\mathrm{pkt}}, A_{\mathrm{src}}\right)$. Each node also maintains a history table $\mathbb{H}$ to store all seen pairs. Then, a node can identify duplicate packets by checking $P$. So the second forwarding criterion is that if $P \notin \mathbb{H}$, this packet is allowed to be forwarded.

In many industrial applications, outdated packets are useless for control systems. Thus, the final forwarding criterion of the proposed scheme is that the packet's age should be checked at every hop and outdated packets should be dropped.

Finally, with all these criteria, all nodes are able to communicate with the gateway by flooding in a controlled manner for both uplink and downlink. More details of REALFLOW can be found in the previous work [8].

\section{Example of REALFLOW}

Fig. 2 illustrates an example of REALFLOW, where $K_{\max }$ is set to be 2. In Fig. 2(a), the propagation of list-update messages is demonstrated. Fig. 2(b) shows node 3 sending a list-response message to the gateway. Node 3 selects node 1 and 2 as its parents. When node 1 and 2 receive list-response messages from node 3 , they add node 3 to their $\mathbb{L}$ and forward the message to the gateway. The dashed line indicates that list-response messages from node 3 will be dropped by node 4 , since node 4 is not a parent of node 3. Fig. 2(c) shows the schedule distribution stage for node 3 . There are two available paths from the gateway to node 3 . Any of these paths can be chosen for schedule distribution. The uplink and downlink are exhibited in Fig. 2(d) and (e). Node 1 and 2 will assist with packet forwarding from/to node 3 , while node 4 will not participate in the packet forwarding. The dash-dotted line means that this transmission might be dropped. For instance, if node 1 has already got a packet from node 3 , then the same packet from node 2 will be dropped. However, if node 1 fails to receive any packet from node 3 , the packet from node 2 will still be forwarded.

The overhead of the network management can be calculated to show the important enhancement. We assume that the network is updated every $15 \mathrm{~s}$ and the refresh rate of uplink and downlink is 1 . Then, we assume that node 3 changes its parent, so all four nodes need to be rescheduled. According to our command distribution method, each node needs at least three messages to activate the decision. Since node 1, 2, 3, and 4 need $1,1,2$, and 2 timeslots, respectively, to receive commands from the $\mathrm{GW}$, the total number of required messages to change the schedule can be calculated as $(1+1+2+2) \times 3=18$. So if one node changes its parent node every $15 \mathrm{~s}$, we need at least 18 messages to update the whole network. During this $15 \mathrm{~s}$, since the refresh rate of each node is one packet per second, and nodes $1,2,3$, and 4 need 2, 2, 3, and 3 timeslots per second for data transmission, the total number of data transmissions within $15 \mathrm{~s}$ : $(2+2+3+3) \times 15=150$. Thus, the minimum overhead for the network management is about $10.7 \%$. Therefore, if we can 


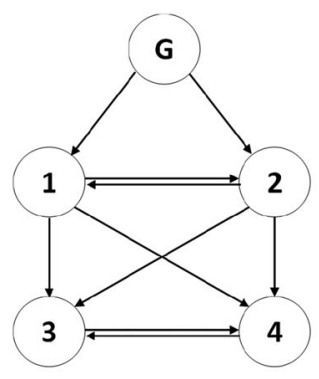

(a)

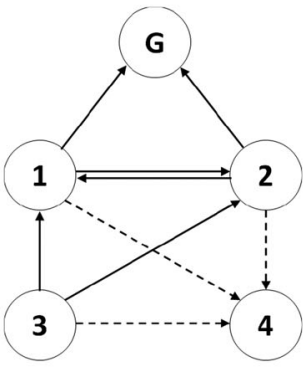

(b)

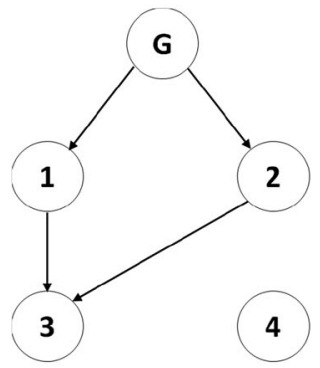

(c)

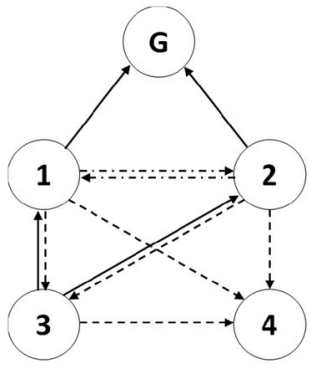

(d)

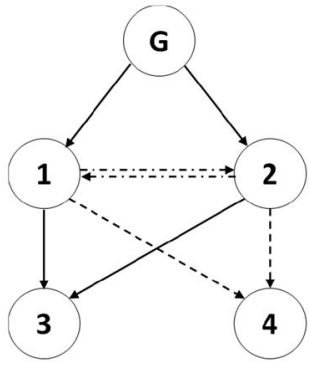

(e)

Fig. 2. Example of REALFLOW: network size $=5, K_{\mathrm{max}}=2$, target node: node 3, solid arrow: accepted transmission, dash arrow: dropped transmission, dash-dotted arrow: conditional accepted transmission. (a) List-update message delivery. (b) List-response message delivery. (c) Schedule distribution. (d) Uplink transmission. (e) Downlink transmission.

TABLE III

Protocol PARAMETERS

\begin{tabular}{ll}
\hline \hline Parameter & \multicolumn{1}{c}{ Description } \\
\hline Application layer refresh interval & Constant bit rate application 1 s \\
Network layer & flooding-based routing protocols \\
MAC layer timeslot duration & TDMA $10 \mathrm{~ms}$ \\
Bit rate channel frequency & $250 \mathrm{kbps} 2.4 \mathrm{GHz}$ \\
Fading model shadowing model transmit power & Rayleigh fading Log-normal $10 \mathrm{dBm}$ \\
\hline \hline
\end{tabular}

prevent the unnecessary parent change by the enhanced parent selection method, at least $10.7 \%$ of the network traffic for the network management can be decreased.

\section{SIMULATIONS AND ANALYSIS}

In this section, REALFLOW is compared with four floodingbased routing protocols via simulations. The experimental scenarios are described, followed by the analysis of the simulation results.

\section{A. Simulation Settings}

The protocol stack is built in QualNet, and REALFLOW is implemented on the network layer, as well as four other floodingbased routing protocols: Normal flooding, location-based flooding [22], directional flooding [20], and single gossiping with directional flooding (SGDF) [21]. The configurations of Qualnet are summarized in Table III. Nonline-of-sight communication dominates in many industrial environments [24], so the Rayleigh fading model is chosen and the log-normal model is selected as the shadowing model.

\section{B. Evaluation Criteria}

Three characteristics are essential in order to evaluate the performance of REALFLOW: 1) packet delivery ratio within deadlines (PDRD): received packets at destinations before deadlines, where deadline equals the packet refresh interval; 2) consecutive packet loss (CPL): the duration of continuous failures; 3) total packet transmissions: the total amount of packets being sent by the source and intermediate nodes.
PDRD reveals the reliability performance for deadline constrained applications. However, PDRD does not provide sufficient confidence with respect to availability. Several long burst errors can still trigger failures of applications. Thus, the length of CPL are measured. Transmission efficiency is also analyzed by calculating total transmission times.

\section{Simulation Scenarios}

1) Scenario I: Since other flooding-based protocols can only support uplink, downlink is not evaluated in this scenario. For REALFLOW, we let $K_{\max }$ to be $2,3 . K_{\max }=1$ is not chosen, since a little multipath diversity can be provided; if $K_{\max }$ is larger than 3, the network may become unschedulable. In this scenario, a random network topology is created. The gateway Node 1 is placed in the center of the network, and eigth sensor nodes are randomly distributed. Each node periodically sends packets to the gateway. The reason why we included eight nodes in this scenario is because we intend to map the simulation results to the measurement results, which will be describe later. Moreover, the number of nodes in IWSANs used in a process automation cannot be large; otherwise, the network congestion may occur and the required refresh rates cannot be met. Finally, uplink PDRD, CPL, and total packet transmissions are measured.

2) Scenario II: In this scenario, REALFLOW $\left(K_{\max }=\right.$ $2,3)$ is compared with normal flooding, and both uplink and downlink are evaluated. Four sensor nodes and four actuator nodes are included, and uplink/downlink PDRD, CPL, and total packet transmissions are measured.

3) Scenario III: In this scenario, the constraint in terms of the network size is investigated. The network size is increased from 4 to 50 and the PDRD of normal flooding and REALFLOW ( $\left.K_{\max }=2,3\right)$ is measured. Half of the nodes are sensors, the other half are actuators. Each simulation for one network size lasts for $600 \mathrm{~s}$. To obtain more general results, each result is averaged over ten random topologies.

\section{Simulation Results and Analysis}

This section describes the simulation results in all scenarios, followed by the analysis of the simulation results. 


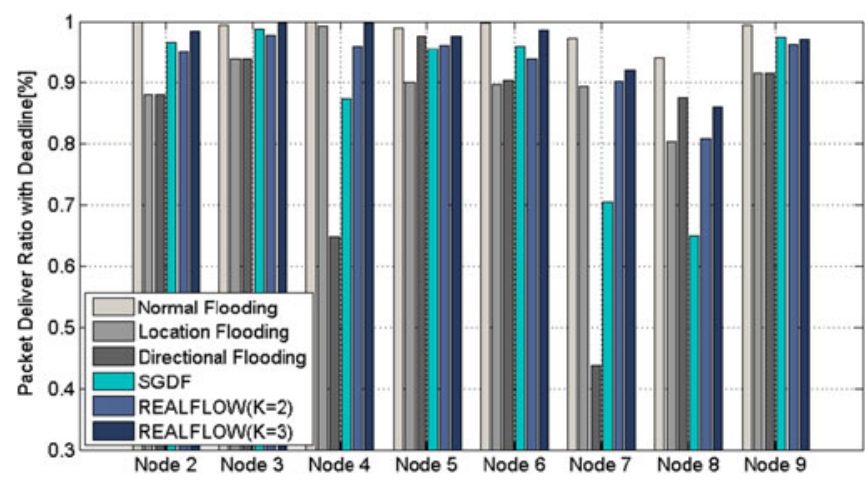

Fig. 3. PDRD of all nodes in Scenario 1 (uplink only, eight nodes).

1) Scenario l: The uplink PDRD of all nodes are illustrated in Fig. 3. It is obvious that normal flooding performs better than the rest due to more transmission paths. Since one timeslot duration is $10 \mathrm{~ms}, 1 \mathrm{~s}$ contains 100 timeslots. If one packet is forwarded by any node only once, each packet needs at most eight timeslots, which equals to the total number of nodes. As each node sends one data packet per second to the gateway, the maximum required timeslot for normal flooding per second is $8 \times 8=64$, which is less than 100 timeslots. Thus, there are sufficient timeslots for normal flooding. Because there is not network congestion, all nodes participate in packet forwarding by normal flooding, so the PDRD of normal flooding can be considered as the upper bound [2]. REALFLOW with $K_{\max }=3$ has on average the second best performance, which is very close to normal flooding for more than half of the nodes. When $K_{\max }$ is changed to 2, PDRD becomes worse due to fewer propagating paths. Location flooding also achieves decent performance, but still worse than REALFLOW. For location flooding, a predefined distance threshold is used to filter out additional forwarding. Because the distance between any nodes is quite random, this unified threshold may block some available paths. The rest of the routing protocols have different reliability performance in different nodes. The reason can be explained as follows. Directional flooding only uses hop number as gradient to assist packet forwarding. Depending on the network topology, the multipath diversity for different nodes may vary a lot. The SGDF protocol also applies a threshold to constrain forwarding redundancy. A random number in each node is generated and compared with the threshold to decide whether or not to forward packets. Therefore, packet forwarding in different nodes may behave differently.

In Fig. 4, six histograms show the frequency distribution of consecutive lost packets. Shorter lengths of consecutive errors indicate lower risks of application halt. It is notable that this result corresponds to the previous one. The protocol with higher PDRD has shorter length of consecutive errors. Thus, normal flooding still performs the best, and REALFLOW with $K_{\max }=3$ is again the second best. When $K_{\max }$ decreases, more and longer consecutive errors occur. Directional flooding is at the bottom of the performance evaluation. Since the longest consecutive failure is up to eight packets, most of industrial control systems cannot tolerate such long packet losses.
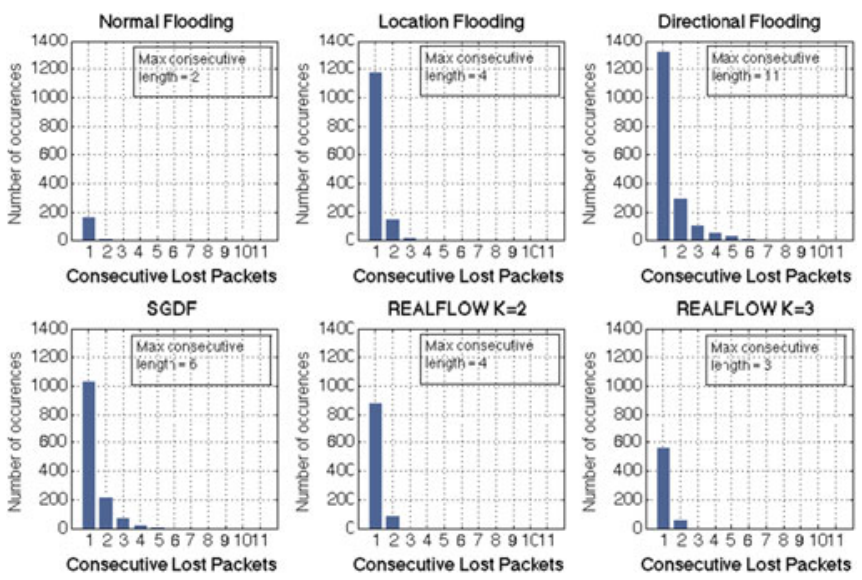

Fig. 4. Distribution of lost packets or not received within deadlines in Scenario 1 (uplink only, eigth nodes).

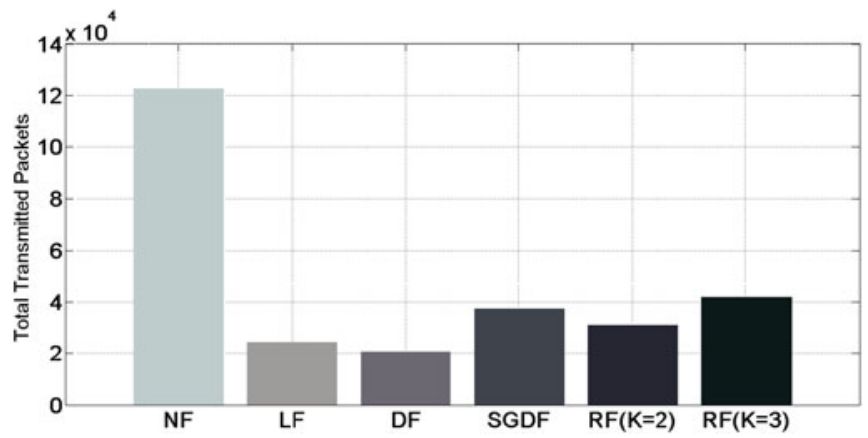

Fig. 5. Total network packet transmissions in Scenario 1 (uplink only, eight nodes, NF = normal flooding, LF = location flooding, DF = directional flooding, $\mathrm{RF}=$ realflow).

The total transmitted packets is shown in Fig. 5. It is obvious that the total number of packet transmissions for normal flooding is much higher than the rest due to excessive packet forwarding. For REALFLOW with $K_{\max }=3$, each node chooses more parent than that with $K_{\max }=2$, but the increment of total packet transmissions is only about $20 \%$. One reason could be that not all nodes can find $K_{\max }$ parents due to bad link quality to neighbors. Thus, the increment of parent nodes may not trigger a sharp growth of the packet forwarding. The number of packets forwarded from directional flooding is the smallest, because only using hop number as gradient gives constrained multipath diversity, which limits packet forwarding. Location flooding also have a small number of transmissions. The number of transmissions from SGDF is the third highest, because SGDF combines the single gossiping and directional flooding, and its forwarding criteria does not depend on the channel and network status, but on random numbers, which may cause excessive forwarding.

2) Scenario II: Average uplink/downlink PDRD for normal flooding and REALFLOW are shown in Fig. 6. The histograms of the frequency distribution of lost packets are illustrated in Fig. 7, and the total number of packet transmissions are summarized in Fig. 8. Similarly to the previous scenario, normal flooding has the highest PDRD and shortest length of 


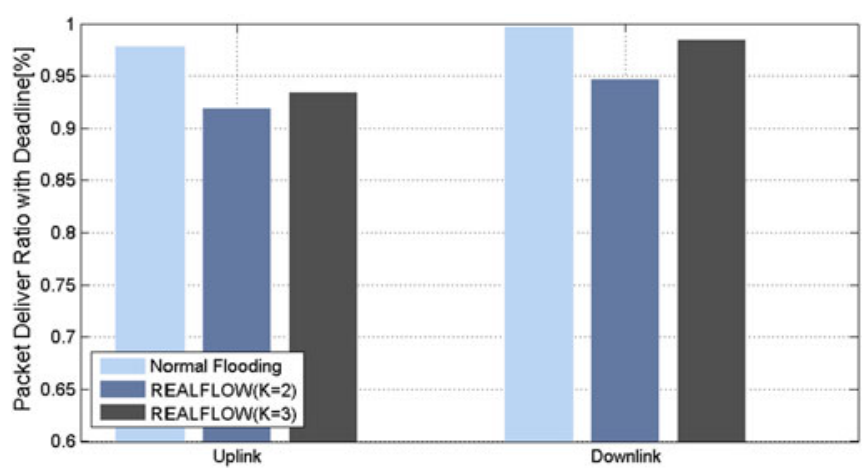

Fig. 6. PDRD in Scenario 2 (uplink and downlink, eight nodes).
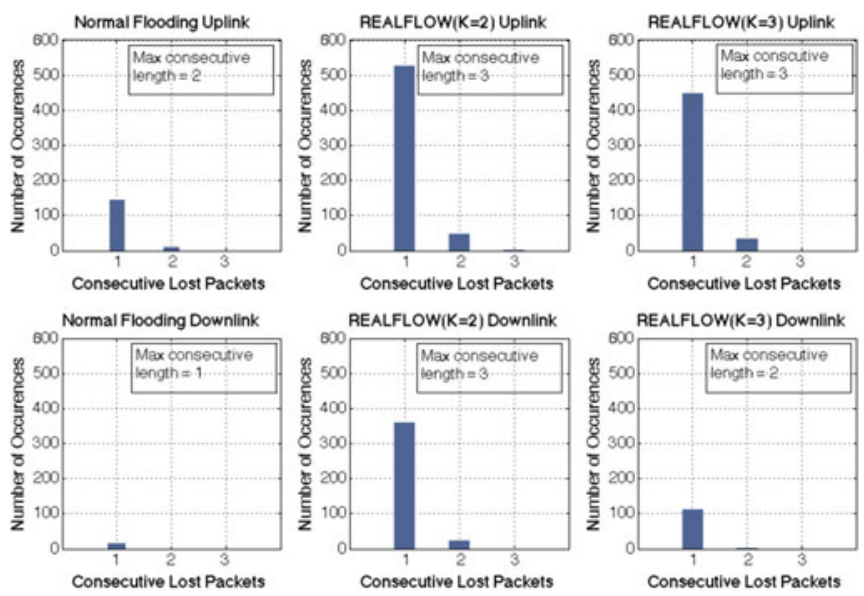

Fig. 7. Distribution of lost packets or not received within deadlines in Scenario 2 (uplink and downlink, eight nodes).

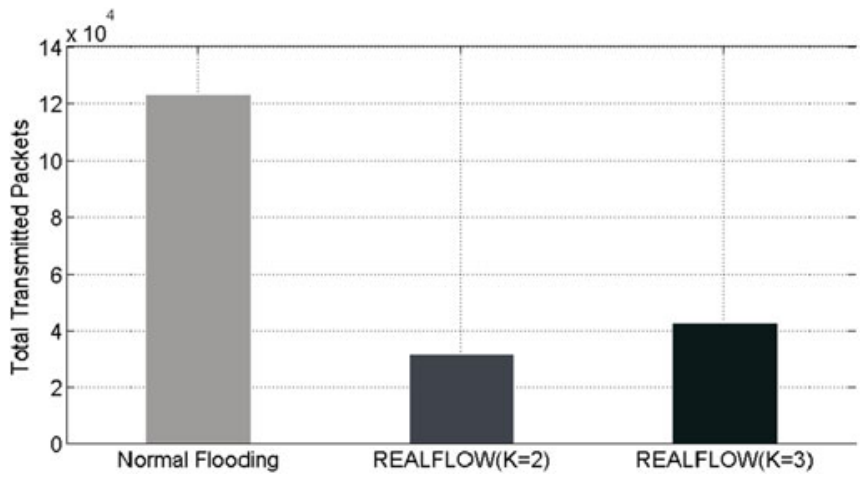

Fig. 8. Total network packet transmissions in Scenario 2 (uplink and downlink, eightnodes).

consecutive errors at the cost of transmission redundancy. The number of transmissions of normal flooding is about four times more than REALFLOW with $K_{\max }=2$ and three times more than $K_{\max }=3$. However, the PDRD of $K_{\max }=3$ is only $5 \%$ less than the normal flooding for the uplink and $2 \%$ for the downlink. Excessive transmission redundancy leads to low network resource efficiency for normal flooding. REALFLOW with $K_{\max }=2$ also performs decently. It reduces the total packet transmissions by $25 \%$, when comparing with $K_{\max }=3$.

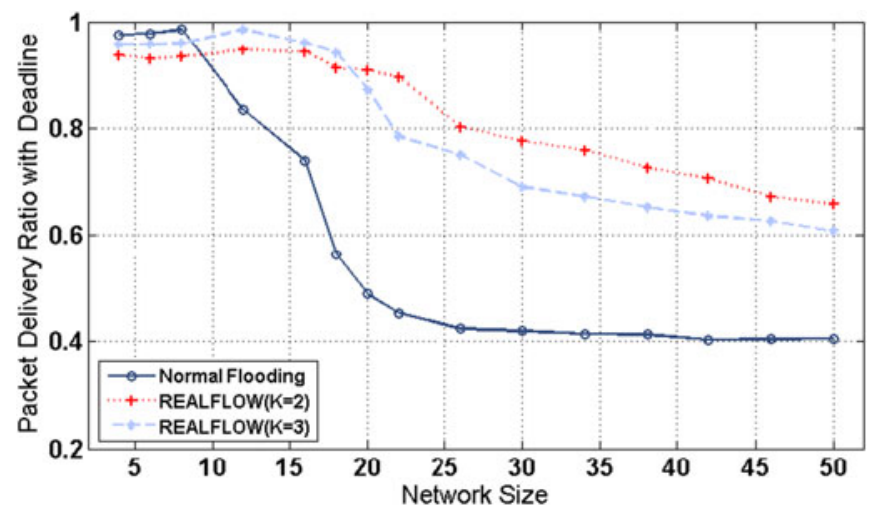

Fig. 9. Average PDRD versus network size in Scenario 3.

Depending on the practical applications, for instance, if the system can be tolerant of four consecutive errors, REALFLOW with $K_{\max }=2$ may still be a potential candidate.

3) Scenario III: Fig. 9 shows the dependence of PDRD on the network size. The results indicate that from the network size 4 to 8 normal flooding performs the best in reliability, because no network congestion occurs. When the network size is less than 8 , maximum multipath diversity can be achieved. When the network size is greater than 8, the PDRD drops drastically. Moreover, when the network size grows to 22, the network is seriously congested. Therefore, after the network size is 22 , the decrease of PDRD becomes less. The PDRD may be even lower for larger network size, but it is out of our interest, since after the network size is larger than 8, normal flooding cannot be used due to serious traffic congestion. It is also notable that although normal flooding performs better than REALFLOW for small network sizes, a great amount of packet transmissions are demanded by normal flooding according to previous simulation results.

For REALFLOW, from the network size 4 to 12 , both PDRD of $K_{\max }=2$ and 3 are larger than 0.9. The result also shows that for these network sizes, the PDRD of $K_{\max }=3$ is better than $K_{\max }=2$ due to more transmission paths. However, when the network size is larger than 12, network congestion starts to appear for $K_{\max }=3$ and its PDRD starts to drop, but it is still higher than $K_{\max }=2$ until the network size is increased to 18 . The network congestion for $K_{\max }=2$ appears at a network size of 16 , later than $K_{\max }=3$, because when the network size increases, the exhaustion of timeslots comes earlier for larger $K_{\max }$. From the figure, it is also notable that when the network gets congested, the larger $K_{\max }$ is, the more drastically the reliability decreases. Therefore, for network sizes above 16 , the PDRD of $K_{\max }=3$ drops more drastically and is lower than that of $K_{\max }=2$. When the network size continues to increase, the PDRD of both $K_{\max }=2$ and $K_{\max }=3$ may also reach 0.4, but it is also out of our interest, since REAFLOW for $K_{\max }=2$ and $K_{\max }=3$ cannot be used when the network size increase to 16 and 12, respectively, due to network congestion.

Therefore, the conclusion is that when the network size is smaller than 8 and high reliability is the biggest concern rather than network efficiency, normal flooding may be considered; 


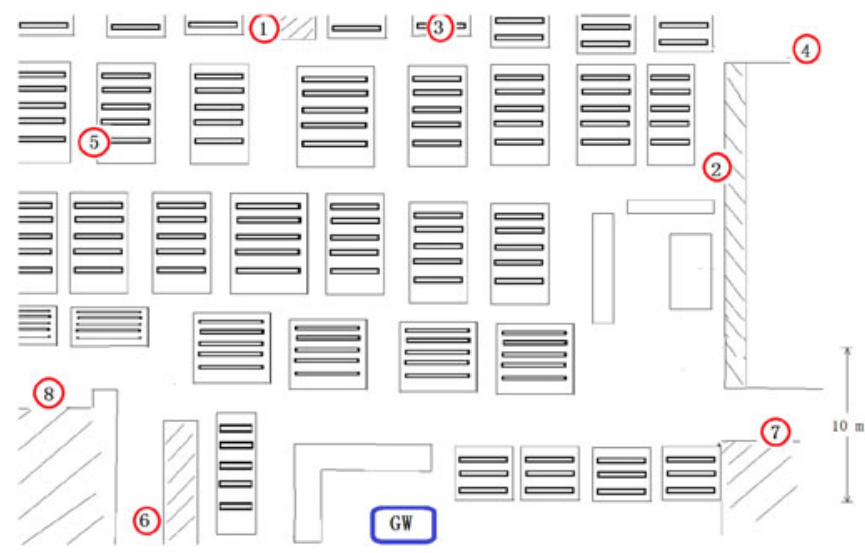

Fig. 10. Node deployment position in the manufacturing workshop, red circle: sensor/actuator nodes, purple rectangle: gateway.

otherwise, REALFLOW with $K_{\max }=3$ is the better option, until the network size reaches 16 .

\section{IMPLEMENTATION AND MEASUREMENTS}

An IWSAN prototype has been built to investigate the performance of REALFLOW in real industrial environments. In this section, the prototype implementation is introduced, followed by the description of the measurements in an industrial manufacturing workshop. Finally, the measurement results are analyzed to exhibit the performance of REALFLOW.

\section{A. System Implementation}

Two hardware platforms are used in the system implementation: STM32W-SK and STM3240G from ST Electronics. The first platform is used to build sensor and actuator nodes, as well as the radio component of the gateway. The gateway is the combination of two platforms, and an RS232 interface is used to connect two platforms.

The complete protocol stack is built in the prototype. The physical layer is based on the functions provided by the radio chipset. The TDMA is implemented with the timeslot duration $10 \mathrm{~ms}$. The Gateway maintains a time source module to manage network-wide time synchronization. All nodes synchronize themselves with the gateway.

\section{B. Measurement Settings}

The experiment was conducted in an industrial manufacturing workshop. It is worth mentioning that few researchers can get access to real industrial environments due to safety reasons in hazardous areas during full production speed. We are fortunate to conduct the measurement in this industrial manufacturing environment for a short period. Although the inner space of the workshop is large without many concrete walls, a great deal of metallic equipment exists. In our measurement, one gateway and eight nodes were randomly deployed. The positions of the gateway and all nodes are shown in Fig. 10, where node 1, 2, 3 , and 4 are set to be sensor nodes, and node 5, 6, 7, and 8 are

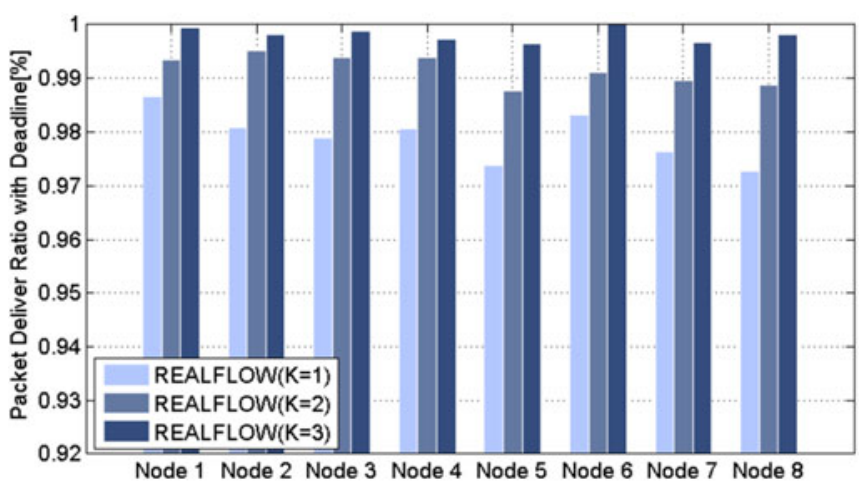

Fig. 11. PDRD of all nodes in measurement (uplink and downlink, eight nodes).

configured as actuator nodes. The range from nodes to the GW is from 15 to $40 \mathrm{~m}$.

During the measurement period, the manufacturing activities did not stop. The metallic equipment was moved by forklifts all the time. Thus, the environment is not static. Before the measurement, a spectrum analyzer was used to check if there are other in-band signals from other wireless systems, such as WLAN or Bluetooth. In [25], Sikora and Groza pointed out the impacts from other wireless systems using ISM band on IEEE 802.15.4 may increase the packet error rate. Therefore, we conducted the background noise measurement for over 10 min, and the maximum peak (MaxHoldPeak) values were measured. The frequency for measurement was $2.47 \mathrm{GHz}$, where the maximum peak values of background noise was $-53 \mathrm{dBm}$, indicating the existence of other wireless systems in the same area. If the distance between those of our field devices and WLAN routers or Bluetooth devices are short, those in-band signals from other wireless systems will have the negative impact on our packet delivery. The enhancement of route management and flooding-based mechanism of REALFLOW may avoid application failures. So one of the purposes of this measurement is to investigate to what extent those interferences impact on our prototype.

The refresh rates for sensors and actuators are 1 packet per second. The gateway broadcasts list-update messages every $15 \mathrm{~s}$ for network maintenance. $K_{\max }$ is set to be 1,2 , and 3 , respectively, and measurements for each $K_{\max }$ were only conducted for around one hour due to the limited access time. PDRD was measured at both sensor and actuator sides. However, in light of the limited memory footprint, other measurements for actuators are currently unavailable.

\section{Measurement Results and Analysis}

The PDRD of all eight nodes is illustrated in Fig. 11. For all nodes, when the parameter $K_{\max }$ increases, PDRD also increases. It is notable that in most of the nodes, the PDRD performance improvement from $K_{\max }=1$ to $K_{\max }=2$ is more significant than that from $K_{\max }=2$ to $K_{\max }=3$. The reason can be explained mathematically as follows. It is assumed that there are three available paths from a node to its three parent nodes, with the PDR $p_{1}, p_{2}$, and $p_{3}$, respectively 

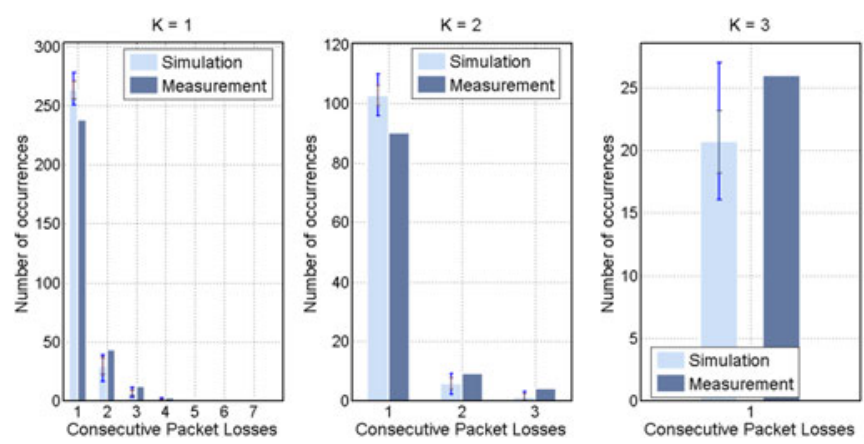

Fig. 12. Distribution of lost packets or not received within deadlines in measurement (uplink and downlink, eight nodes).

$\left(0 \leq \mathrm{p}_{1} \mathrm{p}_{2} \mathrm{p}_{3} \leq 1\right)$. When $K_{\max }$ increases, more parents will be selected. $P_{k 1}, P_{k 2}$, and $P_{k 3}$ represent the PDR when $K_{\max }=1,2,3$. Then, $P_{k 1}=p_{1}, P_{k 2}=p_{1}+\left(1-p_{1}\right) p_{2}$, and $P_{k 3}=p_{1}+\left(1-p_{1}\right) p_{2}+\left(1-p_{1}\right)\left(1-p_{2}\right) p_{3}$. Then, $\left(P_{k 3}-\right.$ $\left.P_{k 2}\right)-\left(P_{k 2}-P_{k 1}\right)=\left(1-p_{1}\right)\left(\left(1-p_{2}\right) p_{3}-p_{2}\right)$. According to the measurement results, $p_{2}$ is very likely larger than 0.5 , so $\left(1-p_{2}\right) p_{3}<0.5$. Finally, it can be computed that $\left(P_{k 3}-P_{k 2}\right)<\left(P_{k 2}-P_{k 1}\right)$. Therefore, the measurement results correspond to the theoretical analysis in a proper way.

The histograms of the frequency distribution of lost packets for uplink are illustrated in Fig. 12. These results confirm that higher reliability brings shorter consecutive errors. Eleven continuous errors occur when $K_{\max }=1$, which might cause by temporary signal blocking from the moving forklifts or workers. When $K_{\max }$ increases, the length of the maximum CPL significantly decreases. The explanation is that due to more alternative paths, even if one path is blocked by obstacles, the packets can bypass the obstacles through another path, but there are still three continuous errors. It indicates the transmission signal might be blocked or interfered in all paths. When $K_{\max }$ increases to 3, no consecutive error is seen during the measurement period.

Since the measurement time was limited as we emphasized before, even if no consecutive error is observed when $K_{\max }=3$, it is difficult to make any conclusion that by using $K_{\max }=3$, consecutive errors will never occur for any longer time. Thus, the measurement is further compared with the simulation results to investigate the correlation and verify the rationality. A similar scenario is created in QualNet, and the simulation time is $24 \mathrm{~h}$. To compare with the measurement results, simulation results are collected per hour, same as the measurement time. Then, the mean value, standard deviation, and $\mathrm{max} / \mathrm{min}$ of consecutive errors are calculated based on the results collected each hour, which is plotted in Fig. 12. According to the figure, consecutive errors from the measurements follows a similar trend as those from simulations. On average, more single errors are seen in simulation, while measurement results have more consecutive errors. The reason is that the movement of forklifts and workers causes deep fading of the wireless signals. Moreover, other wireless systems using the same frequency will also interfere with our signals. Both these factors will cause longer consecutive errors in reality. According to the comparison, the measurement results still correspond to the simulations in a good way, which indicates the simulation results to be reasonable. This
TABLE IV

ApPlication FaILURE Probability Estimation

\begin{tabular}{lccc}
\hline \hline \multicolumn{4}{c}{$(2,3)$-firm deadlines } \\
\hline & Measured One hour & Estimated One Day & Estimated One week \\
\hline$K_{\max }=1$ & 0.0063 & 0.1407 & 0.6541 \\
$K_{\max }=2$ & 0.0009 & 0.0213 & 0.1403 \\
$K_{\max }=3$ & - & - & - \\
\hline
\end{tabular}

comparison also shows that the dynamic environment and those in-band signals from other wireless systems do have impacts on the performance of our protocol, but the consecutive packet errors from simulation and measurement are still comparable.

To further visualize the improvement, the application failure probability is investigated. In industrial automation, applications may fail due to burst errors. A (2, 3)-firm deadline is often used in industrial automation, where the loss of at most two out of three consecutive packets is deemed acceptable. Therefore, based on the measurement results, the probability of three consecutive errors for different $K_{\max }$ is calculated in the nodes having the most consecutive errors. Based on the measurement time, the application failure probability for different lengths of operating time can be extrapolated. All the results are summarized in Table IV. When $K_{\max }=1$, applications are at very high risk of getting terminated in a long term. $K_{\max }=2$ gives much better performance, where the probability of application failure might be lower than $15 \%$ during one week. When $K_{\max }$ increases to 3, the performance is further improved that no application failure is seen for (2, 3)-firm deadline during the measurement period. However, it is difficult to make any estimation for $K_{\max }=3$, because the measurement time only lasts for about $1 \mathrm{~h}$. Although longer measurement periods will give more confidence in the results, under current circumstances, it is impractical to conduct longer measurement in the real industrial environment, but it is obvious that when $K_{\max }$ increases, the application failure probability decreases and availability is improved.

Thus, according to the measurement results and analysis, reliable transmission can be provided by applying REALFLOW. When $K_{\max }=3$, no consecutive errors are seen in earlier measurements or simulations, but it cannot be guaranteed that consecutive errors never appear in this setting. It may bring the confidence of long-term availability, but longer measurement times are required to confirm the performance.

\section{CONCLUSIONS AND FUtURE WORK}

In this paper, the enhanced REALFLOW protocol is proposed for the adaptation to real environments. First, multipath diversity can be achieved by REALFLOW. Second, WBA from the proposed protocol allows packet transmissions not to follow specific paths so that packet forwarding becomes more flexible. Third, it is not only verified by simulation, but also implemented and confirmed in reality. The simulation results show that compared with four other flooding-based protocols, both transmission failures and the length of consecutive errors are decreased by using REALFLOW. Moreover, a prototype has been built, and measurements have been conducted in a real 
industrial manufacturing workshop. The measurement results show that REALFLOW achieves high reliability with deadline constraints and high system availability in a real industrial environment.

Although REALFLOW has several advantages, the limitations cannot be neglected. REALFLOW only support one sink. Since several sinks are often seen in reality, multiple sinks should be supported in the future. Moreover, the parent selection approach needs to be improved with more accurate channel assessment methods, since RSSI cannot reveal the real channel conditions. Furthermore, longer measurements in different industrial environments are also desired to further confirm the performance of our approach, although, in practice, such measurements are very hard to achieve due to production in restricted areas. Low-energy consumption is not the main issue in our settings, but is also a part of the future work.

\section{REFERENCES}

[1] J. Åkerberg, M. Gidlund, and M. Björkman, "Future research challenges in wireless sensor and actuator networks targeting industrial automation," in Proc. IEEE 9th Int. Conf. Ind. Informat., Jul. 2011, pp. 410-415.

[2] A. Willig, "Recent and emerging topics in wireless industrial communications: A selection," IEEE Trans. Ind. Informat., vol. 4, no. 2, pp. 102-124, May 2008.

[3] M. Soyturk and D. Altilar, "Reliable real-time data acquisition for rapidly deployable mission-critical wireless sensor networks," in Proc. IEEE Conf. Comput. Commun., Apr. 2008, pp. 1-6.

[4] C. Caione, D. Brunelli, and L. Benini, "Distributed compressive sampling for lifetime optimization in dense wireless sensor networks," IEEE Trans. Ind. Informat., vol. 8, no. 1, pp. 30-40, Feb. 2012.

[5] J. Ploennigs, V. Vasyutynskyy, and K. Kabitzsch, "Comparative study of energy-efficient sampling approaches for wireless control networks," IEEE Trans. Ind. Informat., vol. 6, no. 3, pp. 416-424, Aug. 2010.

[6] T.-L. Pham and D.-S. Kim, "Routing protocol over lossy links for ISA100.11a industrial wireless networks," Wireless Netw., vol. 20, pp. 2359-2370, 2014.

[7] X. Huang, H. Zhai, and Y. Fang, "Robust cooperative routing protocol in mobile wireless sensor networks," IEEE Trans. Wireless Commun., vol. 7, no. 12, pp. 5278-5285, Dec. 2008.

[8] K. Yu, Z. Pang, M. Gidlund, J. Åkerberg, and M. Björkman, "REALFLOW: Reliable real-time flooding-based routing protocol for industrial wireless sensor networks," Int. J. Distrib. Sens. Netw., vol. 2014, p. 17,2014

[9] M. Masdari and M. Tanabi, "Multipath routing protocols in wireless sensor networks: A survey and analysis," Int. J. Future Gener. Commun. Netw., vol. 6, no. 6, pp. 181-192, Jun. 2013.

[10] P. T. A. Quang and D.-S. Kim, "Enhancing real-time delivery of gradient routing for industrial wireless sensor networks," IEEE Trans. Ind. Informat., vol. 8, no. 1, pp. 61-68, Feb. 2012.

[11] Y. Li, C. S. Chen, Y.-Q. Song, Z. Wang, and Y. Sun, "Enhancing real-time delivery in wireless sensor networks with two-hop information," IEEE Trans. Ind. Informat., vol. 5, no. 2, pp. 113-122, May 2009.

[12] P. T. A. Quang and D.-S. Kim, "Throughput-aware routing for industrial sensor networks: Application to ISA100.11a," IEEE Trans. Ind. Informat., vol. 10, no. 1, pp. 351-363, Feb. 2014.

[13] J. Niu, L. Cheng, Y. Gu, L. Shu, and S. Das, "R3E: Reliable reactive routing enhancement for wireless sensor networks," IEEE Trans. Ind. Informat., vol. 10, no. 1, pp. 784-794, Feb. 2014.

[14] J. Heo, J. Hong, and Y. Cho, "EARQ: Energy aware routing for real-time and reliable communication in wireless industrial sensor networks," IEEE Trans. Ind. Informat., vol. 5, no. 1, pp. 3-11, Feb. 2009.

[15] S. Han, X. Zhu, A. Mok, D. Chen, and M. Nixon, "Reliable and real-time communication in industrial wireless mesh networks," in Proc. IEEE 17th Real-Time Embedded Technol. Appl. Symp., Apr. 2011, pp. 3-12.

[16] Z. Zou, P. Soldati, H. Zhang, and M. Johansson, "Energy-efficient deadline-constrained maximum reliability forwarding in lossy networks," IEEE Trans. Wireless Commun., vol. 11, no. 10, pp. 3474-3483, Oct. 2012.
[17] S. Oh, Y. Yim, J. Lee, H. Park, and S.-H. Kim, "A novel reliable data dissemination protocol for real-time data in wireless sensor networks," in Proc. IEEE Wireless Commun. Netw. Conf., Apr. 2012, pp. 1988-1992.

[18] C. Dombrowski and J. Gross, "Echoring: A low-latency, reliable tokenpassing mac protocol for wireless industrial networks," in Proc. 21st Eur. Wireless Conf., May 2015, pp. 1-8.

[19] Z. Haas, J. Y. Halpern, and L. Li, "Gossip-based ad hoc routing," IEEE Trans. Netw., vol. 14, no. 3, pp. 479-491, Jun. 2006.

[20] L. Zhao, G. Liu, J. Chen, and Z. Zhang, "Flooding and directed diffusion routing algorithm in wireless sensor networks," in Proc. 9th Int. Conf. Hybrid Intell. Syst., Aug. 2009, vol. 2, pp. 235-239.

[21] W. Yen, C.-W. Chen, and C. Hsiang Yang, "Single gossiping with directional flooding routing protocol in wireless sensor networks," in Proc. IEEE 3rd Conf. Ind. Electron. Appl., Jun. 2008, pp. 1604-1609.

[22] F. Barac, J. Akerberg, and M. Gidlund, "A lightweight routing protocol for industrial wireless sensor and actuator networks," in Proc. IEEE 37th Annu. Conf. Ind. Electron. Soc., Nov. 2011, pp. 2980-2985.

[23] F. Ferrari, M. Zimmerling, L. Thiele, and O. Saukh, "Efficient network flooding and time synchronization with glossy," in Proc. 10th Int. Conf. Inf. Process. Sens. Netw., Apr. 2011, pp. 73-84.

[24] J. Åkerberg, M. Gidlund, F. Reichenbach, and M. Björkman, "Measurements on an industrial wireless hart network supporting PROFIsafe: A case study," in Proc. IEEE Conf. Emerging Technol. Factory Autom., Sep. 2011, pp. 1-8.

[25] A. Sikora and V. Groza, "Coexistence of ieee802.15.4 with other systems in the 2.4 GHz-ISM-band," in Proc. IEEE Instrum. Meas. Technol. Conf., May 2005, vol. 3, pp. 1786-1791.

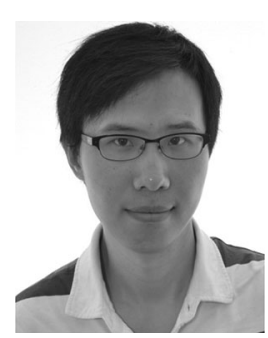

Kan Yu received the B.Sc. degree from Beijing University of Posts and Telecommunications, Beijing, China, in 2005, the M.Sc. degree in communication engineering program from Chalmers University of Technology, Gothenburg, Sweden, in 2010, and the Ph.D. degree in computer science from Mälardalen University, Västerås, Sweden, in 2014.

In 2008, he came to Sweden. From July 2015 to December 2015, he was with the University of Sydney as a Visiting Researcher. Between 2005 and 2008, he was with Huawei Tech., Co., Ltd. and Datang Tele., Co., as an Embedded System Developer. Since 2015, he has been a Postdoctoral Researcher with Mälardalen University. His current research interests include reliable and low latency communications in industrial wireless networks.

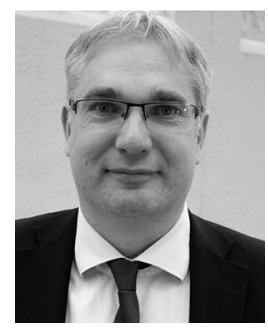

Mikael Gidlund received the Lic.Eng. degree in radio communication systems from the Royal Institute of Technology, Stockholm, Sweden, in 2004, and the Ph.D. degree in electrical engineering from Mid Sweden University, Sundsvall, Sweden, in 2005.

Between 2008 and 2015, he was a Senior Principal Scientist and Global Research Area Coordinator of Wireless Technologies, ABB Corporate Research, Sweden. Between 2007 and 2008, he was a Project Manager and Senior Specialist with Nera Networks AS, Norway. Between 2006 and 2007, he was a Research Engineer and Project Manager with Acreo AB, Sweden. Since 2015, he has been a Professor of computer engineering with Mid Sweden University. He holds more than 20 patents (granted and pending applications) in the area of wireless communication. 


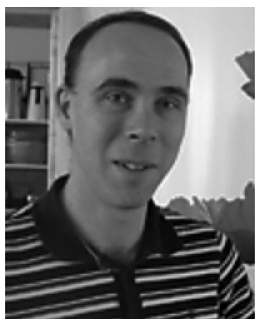

Johan Åkerberg (M'08-SM'11) received the M.Sc. and Ph.D. degrees in computer science and engineering from Mälardalen University, Västerås, Sweden.

$\mathrm{He}$ is currently a Principal Scientist and Global Research Area Coordinator of Embedded Systems and Electronics, ABB Corporate Research, Västerås. He is mainly working with communication for embedded real-time systems in industrial automation and is frequently invited to give talks to governmental bodies, international universities, and automation fairs. He has close to 20 years' experience within $A B B$ in various positions, such as an R\&D Project Manager, Industrial Communication Specialist, and Product Manager. He holds more than ten patents (granted and pending applications) in the area of wired/wireless industrial automation, and is the Author or Coauthor of numerous scientific publications in refereed conferences and journals. He organizes special sessions, hold tutorials, and act as a TCP Member in various distinguished IES conferences.

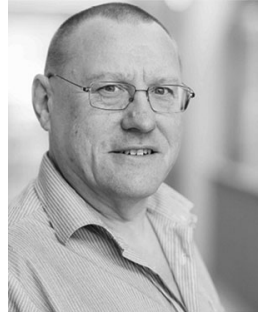

Mats Björkman received the M.Sc. degree in computer science and the Ph.D. degree in computer systems (Datorteknik), the thesis title was Architectures for High Performance Communication, from Uppsala University, Uppsala, Sweden, in 1986 and 1993, respectively.

Between 1993 and 1995, he was a Postdoctoral Researcher with the University of Arizona. In 1995, he returned to Uppsala University as a Senior Lecturer and Researcher in computer communication. During his career, he has during many years between 1988 and 2008 been a part-time Researcher with Swedish Institute of Computer Science. Since 2001, he has been a Professor in computer communication with Mälardalen University, Västerås, Sweden. His research interests include communication performance analysis, wireless communication, and system-wide communication performance and predictability, including safety, security, and other reliability aspects. 